\title{
2D Dubins Path in Environments with Obstacle
}

\author{
Dongxiao Yang, ${ }^{1}$ Didong $\mathrm{Li}^{2}$, and Huafei Sun ${ }^{2}$ \\ ${ }^{1}$ Department of Mechatronical Engineering, Beijing Institute of Technology, Beijing 100081, China \\ ${ }^{2}$ School of Mathematics, Beijing Institute of Technology, Beijing 100081, China
}

Correspondence should be addressed to Huafei Sun; huafeisun@bit.edu.cn

Received 3 June 2013; Revised 14 October 2013; Accepted 15 October 2013

Academic Editor: John Gunnar Carlsson

Copyright (C) 2013 Dongxiao Yang et al. This is an open access article distributed under the Creative Commons Attribution License, which permits unrestricted use, distribution, and reproduction in any medium, provided the original work is properly cited.

We recapitulate the achievement about the Dubins path as well as some precise proofs which are important but omitted by Dubins. Then we prove that the shortest path $\left(R^{*}\right.$-geodesic) in environments with an obstacle consists of no more than five segments, each of which is either an arc or a straight line. To obtain such $R^{*}$-geodesic, an effective algorithm is presented followed by a numerical simulation as verification.

\section{Introduction}

Nowadays, Unmanned Aerial Vehicles (UAVs) are comprehensively applied in a wide range like avionics, GPS-based navigation, flight control techniques, reconnaissance and surveillance, weather, and atmospheric research. In addition, they are also widely used in both conventional combat roles and innovative roles which were not previously possible, such as operations in chemical and biological weapons environments and operations that require microair vehicles [1].

Therefore, one of the most important issues in their development is path planning. A path planning algorithm is aiming at producing one or several flyable paths for UAVs, subject to certain constraints. The path length might be the first factor that should be taken into consideration because the path length decides the traveling time and energy consumption. Besides, the trajectory has to meet the speed and turn limits of the UAVs so that the UAVs could fly safely. Now we can present this problem from the perspective of mathematics.

Given two points $u$ and $v$ in a plane, each with a prescribed direction of motion $U$ and $V$ in it; the question being asked is to find the shortest smooth path of bounded curvature $R$ that joins them. The classical result [2] achieved by Dubins in 1957 gives a sufficient set of paths (each consisting of circular arcs and straight line segments) which always contains the shortest one. Then, Shkel and Lumelsky [3] made classification for the Dubins set, while Chitsaz and Lavalle [4] discussed the directions of the paths, which are more efficient approaches to calculate the shortest path.

For 3D cases, Reeds and Shepp [5] made a "CLC conjecture" in 1990 that was widely believed in the past, which generalized the result for $2 \mathrm{D}$ cases directly to $3 \mathrm{D}$ cases. Unfortunately, such conjecture was disproved by Sussmann in 1995 [6], and he proved that the shortest path might also be a helicoidal arc that has a constant curvature and its torsion satisfies a certain differential equation.

Although such path is exactly the shortest one, it is too complicated to be easily applied in engineering. As a result, researchers began to find other models to get some paths which are easier to be calculated but might be longer than the shortest path. In some papers such as [1, 7-18], the authors considered some approaches to get the final path. One of the advantages of such approaches is that it could be used in the environments with some obstacles only after a little bit changes. A disadvantage is that neither of them is exactly the shortest path.

When it comes to more real and common conditions: UAVs fly through urban environments full of high buildings, and new methods are required to generate trajectories that traverse the regions and its associated obstacles $[1,19]$.

Unfortunately, when considering the Dubins problem with obstacles, the original result will no longer be suitable, because the Dubins path might pass through the obstacles so the result needs to be changed a little. For 3D cases, the approaches used in [8-17] can be used only by a little change, 
because the result is not the shortest paths in either condition. It is a pity that we cannot get the shortest path with obstacles in $3 \mathrm{D}$ cases. Instead, it is possible to find the shortest path with obstacles in $2 \mathrm{D}$ cases. In this paper, we prove that the shortest path consists of no more than five parts, which is either an arc or a straight line under the assumption that there is only one circular obstacle. What is more, both specific algorithm and numerical simulation are presented.

\section{General Case}

In this section, we recapitulate the original proof given by Dubins [2], because this approach is also used in Theorem 13 in Section 3. As a result, parts of them are omitted. On the other hand, the proofs of some details which are very important but not obvious in his paper are omitted. Hence, we give the proofs with the details which are quite useful for the proofs of our results.

Let $P(s)$ be a curve in $\mathbb{E}^{2}$ parameterized by arc length $s$. If

$$
\left\|P^{\prime}\left(s_{1}\right)-P^{\prime}\left(s_{2}\right)\right\| \leq R^{-1}\left|s_{1}-s_{2}\right|
$$

we say that the curve $P$ has an average curvature always less than or equal to $R^{-1}$.

Let $C=C(u, U, v, V, R)$ be the collection of all curves $P$ defined on a closed interval $[0, L]$, where $L=L(P)$ varies with $P$, such that $P(s) \in E^{2}$ for $0 \leq s \leq L ;\left\|P^{\prime}(S)\right\| \equiv 1$; the average curvature of $P$ is everywhere less than or equal to $R^{-1} ; P(0)=$ $u, P^{\prime}(0)=U, P(L)=v$ and $P^{\prime}(L)=V$.

Definition 1. The path which has minimal length in $C=$ $C(u, U, v, V)$ is called the $R$-geodesic for $C$ denoted by $R_{C}$.

Suppose that the arc with radius $R$ is $C$ and the straight line is $L$.

Theorem 2. Every planar R-geodesic is necessarily a continuously differentiable curve which is either (1) CLC or (2) CCC or a subpath of type (1) or (2) (see also Figure 1).

This is the most important theorem in [2] and we present the proofs, beginning with the existence of the $R$-geodesic.

Proposition 3. For any $n, u, U, v, V$, and $R$, there exists a path in $C=C(n, u, U, v, V, R)$ of minimal length.

Proof. Let $l$ be the infimum of lengths of all curves in $C$. There exists a sequence $P_{n} \in C$ such that the length $l_{n}$ is monotonely decreasing to $l$. On one hand, since $\left\|P_{n}^{\prime}\right\| \equiv 1$, it follows that $P_{n}^{\prime}$ are uniformly bounded. On the other hand, since $\left\|P^{\prime}\left(s_{1}\right)-P^{\prime}\left(s_{2}\right)\right\| \leq R^{-1}\left|s_{1}-s_{2}\right|$, it follows that $P_{n}^{\prime}$ are also equicontinuous. Let $\widetilde{P}_{n}$ be the restriction of $P_{n}$ on closed interval $[0, l]$, hence, $\widetilde{P}_{n}$ satisfy all conditions above and are defined on a common compact set $[0, l]$. Then by Ascoli's theorem, there is a subsequence of family $\widetilde{P}_{n}$ whose derivatives converge uniformly on $[0, l]$ to a function $Q$. Then, $\widetilde{P}_{n}$ converge uniformly in $[0, l]$ to a function $P$ with length $l$. It is easy to check that $P$ is exactly the $R$-geodesic $R_{C}$.

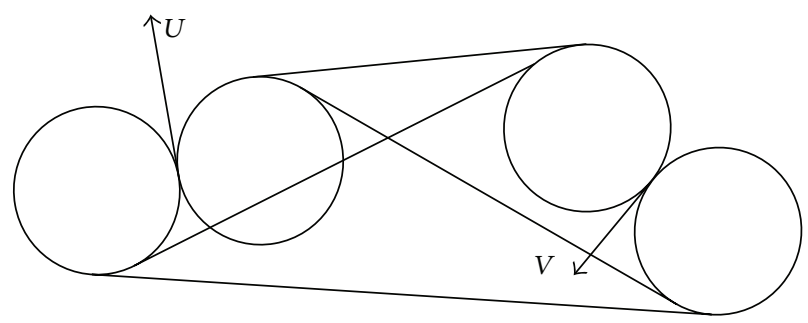

FIGURE 1

Remark 4. The paths $P_{n}$ are defined on different interval $\left[0, l_{n}\right]$, so the Ascoli's theorem could not be applied. To solve this problem, we restrict $P_{n}$ on a common compact interval $[0, l]$ denoted by $\widetilde{P}_{n}$. This step is very important but was omitted by Dubins in [2], so we present it here as a complement.

Then we only relate several propositions without proof.

Proposition 5. Let $P$ be a planar curve of length less than or equal to $\pi R / 8$. Then $P$ is an $R$-geodesic if and only if $P$ is of type CLC.

Then it is easy to prove the following.

Proposition 6. Let $P$ be a planar curve defined on a closed finite interval $[0, l]$ parameterized by arc length. Then if $P$ is a R-geodesic, it is a continuously differentiable curve which consists of a finite number of pieces, each of which is either a straight line segment or an arc of a circle of radius $R$.

In fact, the $R$-geodesic contains no more than three pieces, it is sufficient to show that no path consisting of four arc and straight line segments can be an $R$-geodesic. So we consider all the eight paths of these types: CCCC, CCCL, $C C L C, C L C C, C L C L, L C C C, L C C L$, and LCLC.

Lemma 7. If a curve is an R-geodesic, then so is the curve obtained by traversing the path in the opposite direction.

Therefore, we only need to consider five types: CCCC, CCCL, CCLC, CLCL, and LCCL.

Lemma 8. If a curve is an $R$-geodesic, then so is every subpath of the curve.

Thus, it is sufficient to show that none of the following three types of curves is an R-geodesic: CCCC, CCL, and LCL. Then we consider three lemmas to rule out such cases without proof but only Figures 2, 3, and 4, respectively.

Lemma 9. No curve of type LCL is an R-geodesic.

Lemma 10. No curve of type CCCC is an R-geodesic.

Lemma 11. No curve of type CCL is an R-geodesic. 


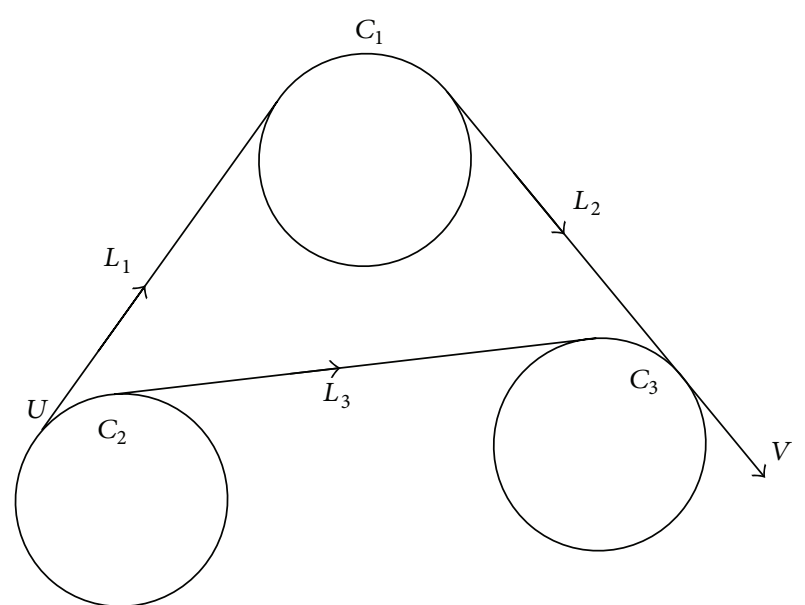

Figure 2

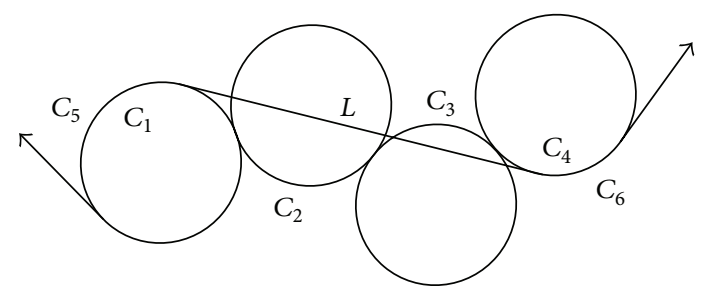

Figure 3

As a result, every planar $R$-geodesic has no more than three pieces except $L C L$, so it is either CCC or CLC or a subpath of them. Now we have proved Theorem 2.

\section{Environments with One Obstacle}

In this section, we consider the path in the environments with one obstacle and prove that the shortest path consists of no more than five segments where each is either an arc or a straight line.

Denote the open circular area by $O$ and we suppose that $R \leq r(O)$ because the radius of obstacle is always far larger than $R$ in practical problems. Suppose that $E^{2 *}$ is the complementary set of $O$ and $D$ is the arc along the bound of $O$. Let $u, v, U$, and let $V$ be vectors in $E^{2}$ and $\|U\|=\|V\|=1$, $R>0$. Let $C^{*}=C^{*}(2, u, v, U, V, R$, and $O)$ be the collection of all curves $P$ in $E^{2 *}$ with an average curvature less than or equal to $R^{-1}$ everywhere.

Definition 12. The shortest path in $C^{*}$ is called the $R^{*}$ geodesic denoted by $R_{C^{*}}$.

If the $R^{*}$-geodesic is the same as $R$-geodesic, we call the obstacle an ineffective obstacle. If they are different, we call it an effective one. Our ultimate goal in this section is to prove the following Theorem 13.

Theorem 13. $R^{*}$-geodesic is one of CCDCC, CLDLC, $C C D L C, C L D C C, C C C, C L C$, or a subpath of them.

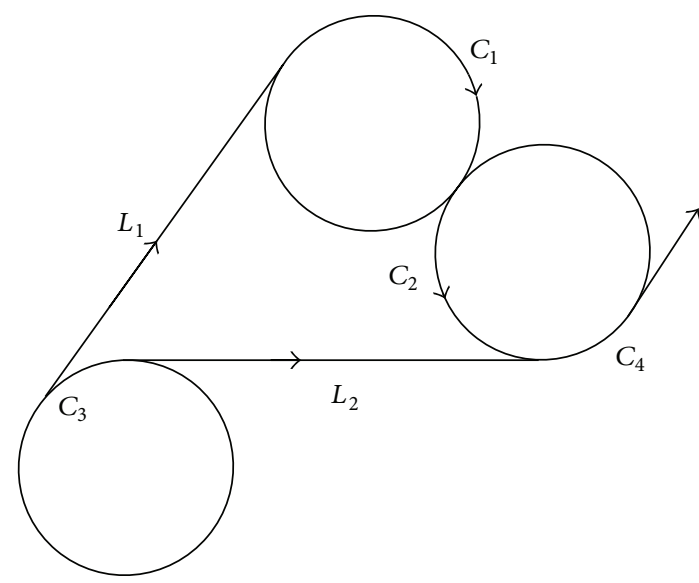

FIGURE 4

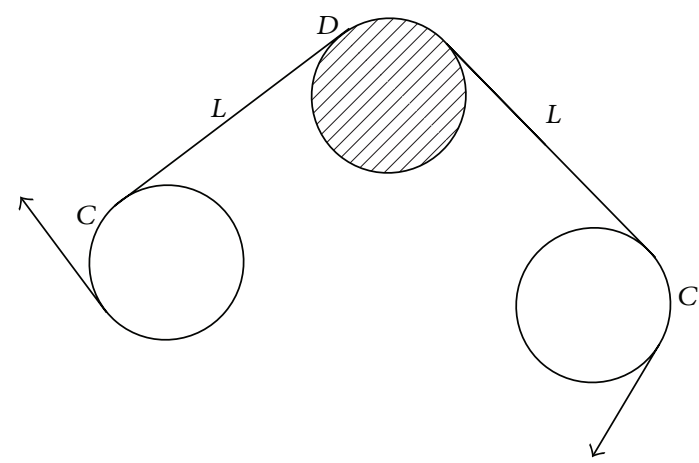

FIGURE 5

Figure 5 is the type CLDLC.

Before proving Theorem 13, we prove several propositions and lemmas first. If the obstacle is ineffective, the $R$-geodesic is showed in Theorem 2, without any difference, so we only consider the case that the obstacle is effective. The obstacle is ineffective means that the $R^{*}$-geodesic is the same as the $R$ geodesic which does not pass through the obstacle $O$. On the other hand, the obstacle in effective means that the original $R$-geodesic passes through the obstacle $O$ such that the new $R^{*}$-geodesic is different.

Proposition 14. For any $n, u, U, v, V$, and $R$, there exists an $P$ in $C^{*}=C^{*}(n, u, U, v, V, R)$ of minimal length.

Proof. As in Proposition 3, such $P_{n}^{\prime}$ are uniformly bounded and equicontinuous. Since $E^{2 *}$ is closed in $E^{2}$, the Ascoli's theorem also holds, so $P_{n}^{\prime}$ converge uniformly to $Q$. Others are the same.

Besides, Propositions 5 and 6 also hold in such case.

Proposition 15. Let $P$ be a planar curve defined on a closed finite interval $[0, l]$ parameterized by arc length. Then if $P$ is an $R^{*}$-geodesic, it is a continuously differentiable curve which consists of a finite number of pieces, each of which is either a straight line segment or an arc of a circle of radius $R$. 
Before we rule out the possibility of a curve which consists of more than five pieces to be the $R^{*}$-geodesic, we prove a lemma.

Lemma 16. If $U$ or $V$ is tangent to the obstacle $O$, then $O$ is ineffective; it is to say that the original $R$-geodesic does not pass through $\mathrm{O}$.

Proof. Theorem 2 shows that the original $R$-geodesic consists of arc or straight lines; both $u$ and $v$ are tangent to the arcs. Since the obstacle $O$ is exactly the bound of the starting circle, $R$-geodesic does not pass through the obstacle, which is shown in Figure 6.

In order to prove Theorem 13, we consider two cases.

\subsection{Case 1: $R_{C^{*}}$ does not intersect $D$}

Proposition 17. $R^{*}$-geodesic is CCC or CLC or subpath of them if it does not contain D.

Proof. It could be proved directly by Theorem 2 .

3.2. Case 2: $R_{C^{*}}$ Intersects $D . R^{*}$-geodesic has several pieces including $D$. Now we prove that there are no more than five pieces.

Proposition 18. $R_{C^{*}}$ has no more than five pieces.

Proof. We prove the proposition by contradiction.

Assume that $R_{C^{*}}$ has six pieces including $D$. Divide $R_{C^{*}}$ into two pieces, with $D$ as the middle piece: the former one is $P_{1} D$, and the latter one is $D P_{2}$.

It is obvious that $P_{1}$ and $P_{2}$ have five pieces altogether; hence, one of them has more than two; pieces. We assume that $P_{1}$ has three pieces and $P_{2}$ has two; see also Figure 7 .

Let the end point of $D$ be $w$; the tangent vector at $w$ is $W$. Now consider $C_{D}^{*}=C(2, u, U, w, W, O)$ and the corresponding $R^{*}$-geodesic $R_{C_{D}^{*}}$. From Lemma 8 , we see that $P_{1} D$ is a subpath of $R^{*}$-geodesic and so is also a $R^{*}$-geodesic: the shortest path for condition $C_{D}^{*}=C(2, u, U, w, W, O)$; hence, $R_{C_{D}^{*}}=P_{1} D$. Besides, $R_{C_{D}}$ is the $R$-geodesic for $C_{D}=$ $C(2, u, U, w, W)$.

Since $W$ is tangent to $O$, we know that $R_{C_{D}^{*}}=R_{C_{D}}$ from Lemma 16, so

$$
R_{C_{D}}=R_{C_{D}^{*}}=P_{1} D
$$

It is to say that the $R$-geodesic $R_{C_{D}}$ for $C_{D}=C(2, u, U, w, W)$ contains four pieces: $P_{1}$ has three and $D$ has one, which is contradict to Theorem 2. Hence, our assumption is wrong and $R_{C^{*}}$ has no more than five pieces.

Now we can prove Theorem 13.

Proof of Theorem 13. Firstly, consider case 2: $R_{C^{*}}$ intersects $D$. From the proof of Proposition 18, especially (2), we know that both $P_{1}$ and $P_{2}$ has no more than two pieces. Thus, $P_{1}$ is CC, $C L, L C$, or subpath of them. Since $P_{1} D=R_{C_{D}}, R_{C_{D}}$ is CCC or CLC or subpath of them from Theorem 2 and

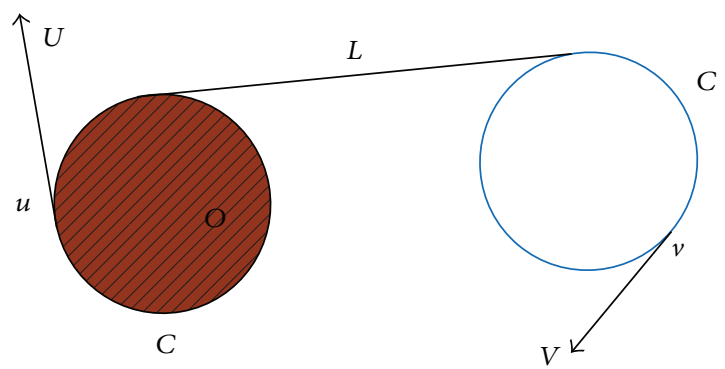

Figure 6

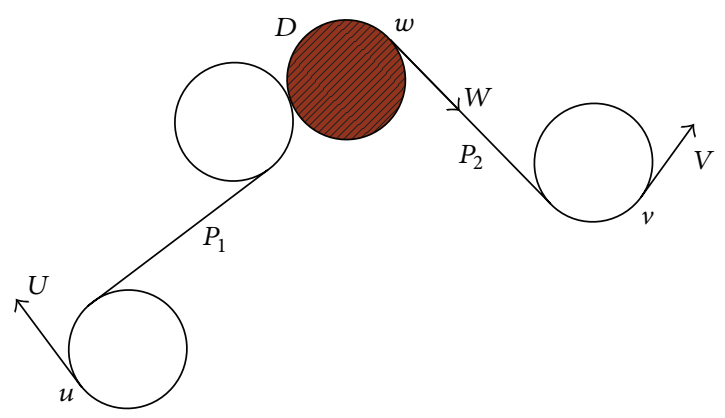

Figure 7

then we can rule out $L C$ easily. Therefore, $P_{1}$ is $C C, C L$, or subpath of them. Similarly, $P_{2}$ is $C C, L C$, or subpath of them. According to Propositions 17 and 18 , the $R^{*}$-geodesic $R_{C^{*}}$ is $C C D C C, C L D L C, C C D L C, C L D C C, C C C, C L C$, or a subpath of them.

Now we have proved Theorem 13 and solved the Dubins problem with obstacle.

\section{Algorithm}

Such Dubins problem mentioned above could be described as input and output. Inputs are as follows:

(1) initial point $P_{s}\left(x_{s}, y_{s}\right)$ and initial velocity $v_{s}$,

(2) final point $P_{f}\left(x_{f}, y_{f}\right)$ and final velocity $v_{f}$,

(3) minimal turning radius of the UAVs $R$,

(4) radius of the circular obstacle $R_{D}$.

While output is the length of the minimizing flyable path 1.

In order to calculate the output with inputs, we present an algorithm which contains seven steps.

Step 1. Calculate the initial turning circle $C_{s}$ by $v_{s}, P_{s}$, and $R$, the final turning circle $C_{f}$ by $v_{f}, P_{f}$, and $R$. Denote the clockwise and counterclockwise circles by $C_{s 1}, C_{f 1}$ and $C_{s 2}, C_{f 2}$, respectively. Then calculate four groups of data according to the original algorithm, while each of them contains a pair of initial and final turning circle, coordinates of two tangent points, and the length of Dubins path.

Step 2. Judge that whether each of the four Dubins path passes through the obstacle circle. 


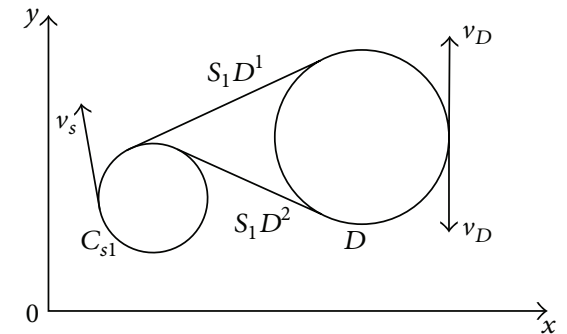

FIGURE 8

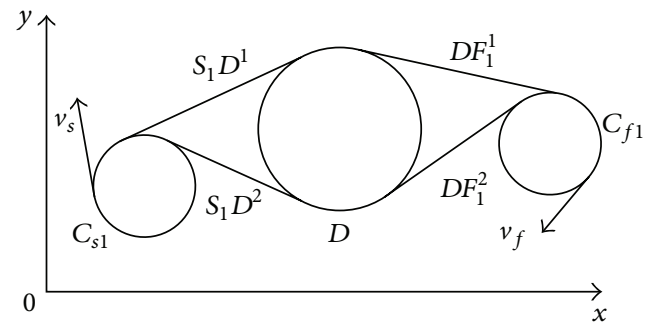

FIGURE 9

Case 1. If the path passes through the obstacle, go to Step 3.

Case 2. Else, record the data and go to Step 7.

Step 3. Calculate the Dubins path between $C_{s 1}$ and obstacle circle $D$. Because the "final" velocity is uncertain, rule out two paths whose turning directions of $v_{s}$ and the common tangent line are different, where the other two remained $S_{1} D^{1}$ and $S_{1} D^{2}$; see also Figure 8.

Step 4. Calculate the Dubins path between obstacle circle $D$ and $C_{f 1}$ by ruling out two of four paths as in Step 3 where $D F_{1}^{1}$ and $D F_{1}^{2}$ remained, as in Figure 9.

Step 5. There are two possible combinations from $C_{s 1}$ to $C_{f 1}$ : $S_{1} D F_{1}^{1}$ and $S_{1} D F_{1}^{2}$, where

$$
S_{1} D F_{1}^{1}=S_{1} D^{1}+D F_{1}^{1} ; \quad S_{1} D F_{1}^{2}=S_{1} D^{2}+D F_{1}^{2} .
$$

Step 6. There are eight combinations in all including $S_{1} D F_{1}^{1}$ and $S_{1} D F_{1}^{2}$. The other six paths are

$$
\begin{aligned}
& S_{1} D F_{2}^{1} \text { and } S_{1} D F_{2}^{2}: \text { from } C_{s 1} \text { to } C_{f 2} \\
& S_{2} D F_{1}^{1} \text { and } S_{2} D F_{1}^{2}: \text { from } C_{s 2} \text { to } C_{f 1} \\
& S_{2} D F_{2}^{1} \text { and } S_{2} D F_{2}^{2}: \text { from } C_{s 2} \text { to } C_{f 2} \text {. }
\end{aligned}
$$

Step 7. Calculate the length of all the eight paths and select the minimal one as the shortest path.

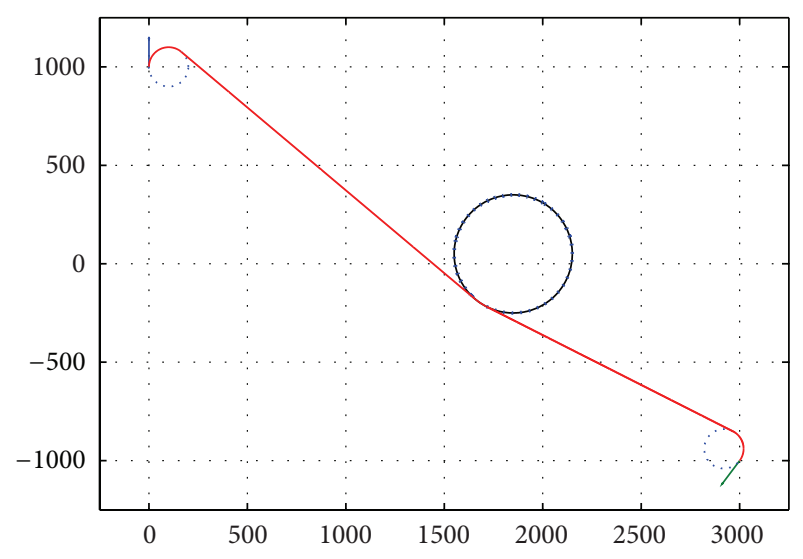

FIgURE 10

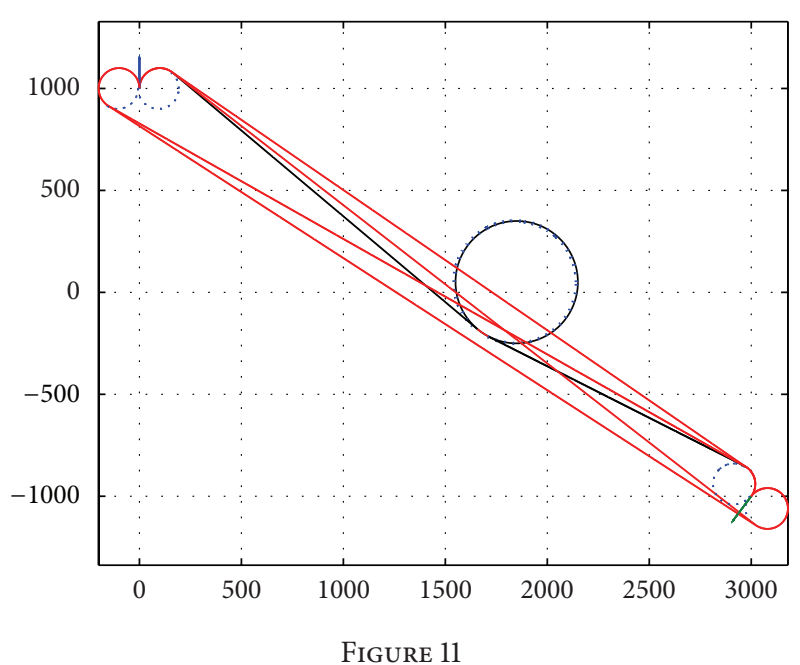

\section{Simulation}

The parameters of UAVs and the obstacle are showed inTable 1.

Since the original path B intersects with the obstacle D, a new path $\mathrm{E}$ which does not intersect $\mathrm{D}$ is calculated, as in Figure 10.

Put E together with three original paths A, C, and D, which do not intersect the obstacle, as in Figure 11.

The lengths of the four paths are shown in Table 2 so that the minimal one E could be selected directly.

\section{Conclusion}

In this paper, we present the achievements of Dubins and his proofs with some more important complement in details at first. Then we generalize the results to the more useful cases in environments with one obstacle and prove that the shortest path $\left(R^{*}\right.$-geodesic) consists of no more than five segments, where each is either an arc or a straight line. To calculate the $R^{*}$-geodesic, an algorithm is discussed and also a numerical simulation is accomplished as verification. 
TABLE 1: Value of parameter.

\begin{tabular}{lcc}
\hline Parameter & Value & Unit \\
\hline Initial coordinates & $(0,1000,200)$ & $\mathrm{m}$ \\
Final coordinates & $(3000,-1000,200)$ & $\mathrm{m}$ \\
Initial velocity & $(0,30)$ & $\mathrm{m} / \mathrm{s}$ \\
Final velocity & $(-18,-24)$ & $\mathrm{m} / \mathrm{s}$ \\
Obstacle coordinates & $(1850,50)$ & $\mathrm{m}$ \\
Obstacle radius & 300 & $\mathrm{~m}$ \\
\hline
\end{tabular}

TABLE 2: Length comparisons.

\begin{tabular}{lcc}
\hline Path & Length & Unit \\
\hline A & 4313.2 & $\mathrm{~m}$ \\
C & 4667.1 & $\mathrm{~m}$ \\
D & 4173.5 & $\mathrm{~m}$ \\
E & 3823.3 & $\mathrm{~m}$ \\
\hline
\end{tabular}

\section{Acknowledgments}

The authors would like to express their sincere thanks to Professor J. G. Carlsson for his valuable suggestions and special thanks to Professor D. Krupka for his help. This subject is supported by the National Natural Science Foundations of China (no. 61179031 and no. 10932002) and National Defense Project NLOS $* * *$ Technology (no. B22 $* * * 0011)$.

\section{References}

[1] M. Shanmugavel, Path planning of multiple autonomous vehicles [Ph.D. thesis], Department of Aerospace, Power \& Sensors, Defence College of Management and Technology, Cranfield University, 2007.

[2] L. E. Dubins, "On curves of minimal length with a constraint on average curvature, and with prescribed initial and terminal positions and tangents," The American Journal of Mathematics, vol. 79, no. 3, pp. 497-516, 1957.

[3] A. M. Shkel and V. Lumelsky, "Classification of the Dubins set," Robotics and Autonomous Systems, vol. 34, no. 4, pp. 179-202, 2001.

[4] H. Chitsaz and S. M. Lavalle, "Time-optimal paths for a Dubins airplane," in Proceedings of the 46th IEEE Conference on Decision and Control, New Orleans, La, USA, 2007.

[5] J. A. Reeds and L. A. Shepp, "Optimal paths for a car that goes both forwards and backwards," Pacific Journal of Mathematics, vol. 145, no. 2, pp. 367-393, 1990.

[6] H. J. Sussmann, "Shortest 3-dimensional paths with a prescribed curvature bound," in Proceedings of the 34th IEEE Conference on Decision and Control, pp. 3306-3312, New Orleans, La, USA, December 1995.

[7] J. J. Kehoe, Trajectory generation for effective sensing of a close proximity environment [Ph.D. dissertation], University of Florida, 2007.

[8] M. Strandberg, "Augmenting RRT-Planners with local trees," in Proceedings of the IEEE International Conference on Robotics and Automation, pp. 3258-3262, New Orleans, La, USA, May 2004.

[9] N. A. Melchior and R. Simmons, "Particle RRT for path planning with uncertainty," in Proceedings of the IEEE International
Conference on Robotics and Automation, pp. 1617-1624, Roma, Italy, April 2007.

[10] J. J. Kuffner Jr. and S. M. LaValle, "RRT-connect: an efficient approach to single-query path planning," in Proceedings of the IEEE International Conference on Robotics and Automation, pp. 995-1001, San Fransisco, Calif, USA, April 2000.

[11] M. Kalisiak and M. van de Panne, "RRT-blossom: RRT with a local flood-fill behavior," in Proceedings of the IEEE International Conference on Robotics and Automation, pp. 1237-1242, Orlando, Fla, USA, May 2006.

[12] D. Ferguson, N. Kalra, and A. Stentz, "Replanning with RRTs," in Proceedings of the IEEE International Conference on Robotics and Automation, pp. 1243-1248, Orlando, Fla, USA, May 2006.

[13] M. Hwangbo, J. Kuffner, and T. Kanade, "Efficient two-phase 3D motion planning for small fixed-wing UAVs," in Proceedings of the IEEE International Conference on Robotics and Automation, pp. 1035-1041, Roma, Italy, April 2007.

[14] J. Z. Sasiadek and I. Duleba, "3D local trajectory planner for UAV," Journal of Intelligent and Robotic Systems, vol. 29, no. 2, pp. 191-210, 2000.

[15] M. Shanmugavel, A. Tsourdos, R. Zbikowski, and B. A. White, "3D Dubins sets based coordinated path planning for swarm of UAVs," in Proceedings of the AIAA Guidance, Navigation, and Control Conference and Exhibit, Keystone, Colo, USA, August 2006.

[16] Y. Kuwata and J. How, "Three dimensional receding horizon control for UAVs," in Proceedings of the AIAA Guidance, Navigation, and Control Conference and Exhibit, Providence, RI, USA, August 2004.

[17] A. J. Pachikara, J. J. Kehoe, and R. Lind, "A pathparameterization approach using trajectory primitives for 3-dimensional motion planning," in Proceedings of the AIAA Guidance, Navigation, and Control Conference, Chicago, Ill, USA, August 2009.

[18] X. Zhang, H. Duan, and Y. Yu, "Receding horizon control for multi-UAVs close formation control based on differential evolution," Science China Information Sciences, vol. 53, no. 2, pp. 223-235, 2010.

[19] R. D. Hurley, Three-dimensional trajectory generation for flight within an obstacle rich environment [M.S. thesis], University of Florida, 2009. 


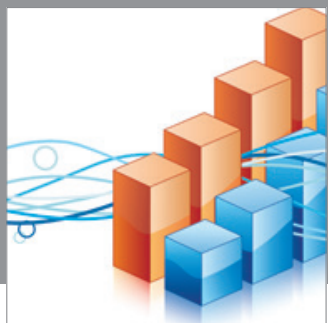

Advances in

Operations Research

mansans

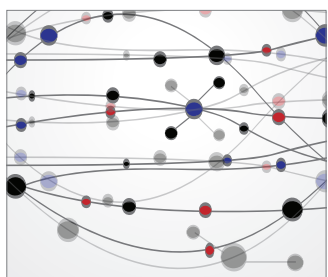

The Scientific World Journal
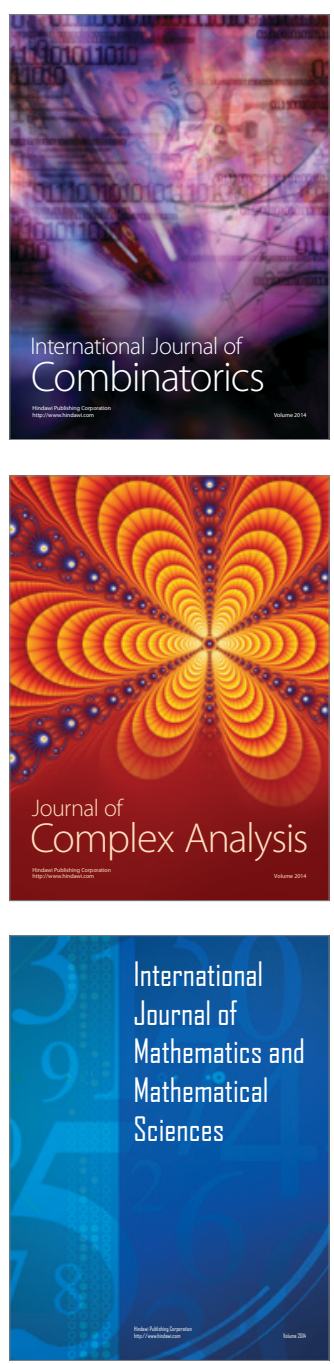
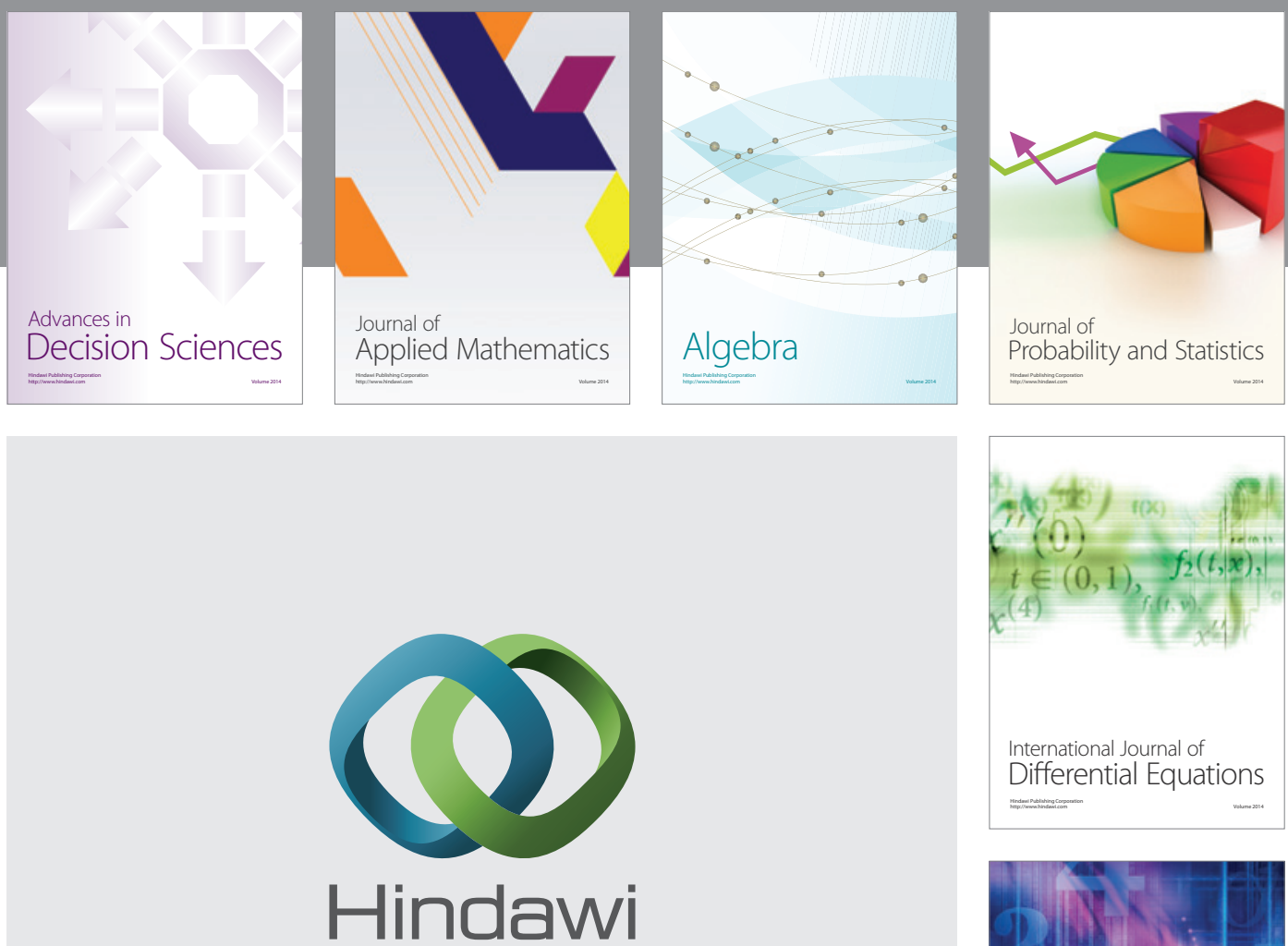

Submit your manuscripts at http://www.hindawi.com
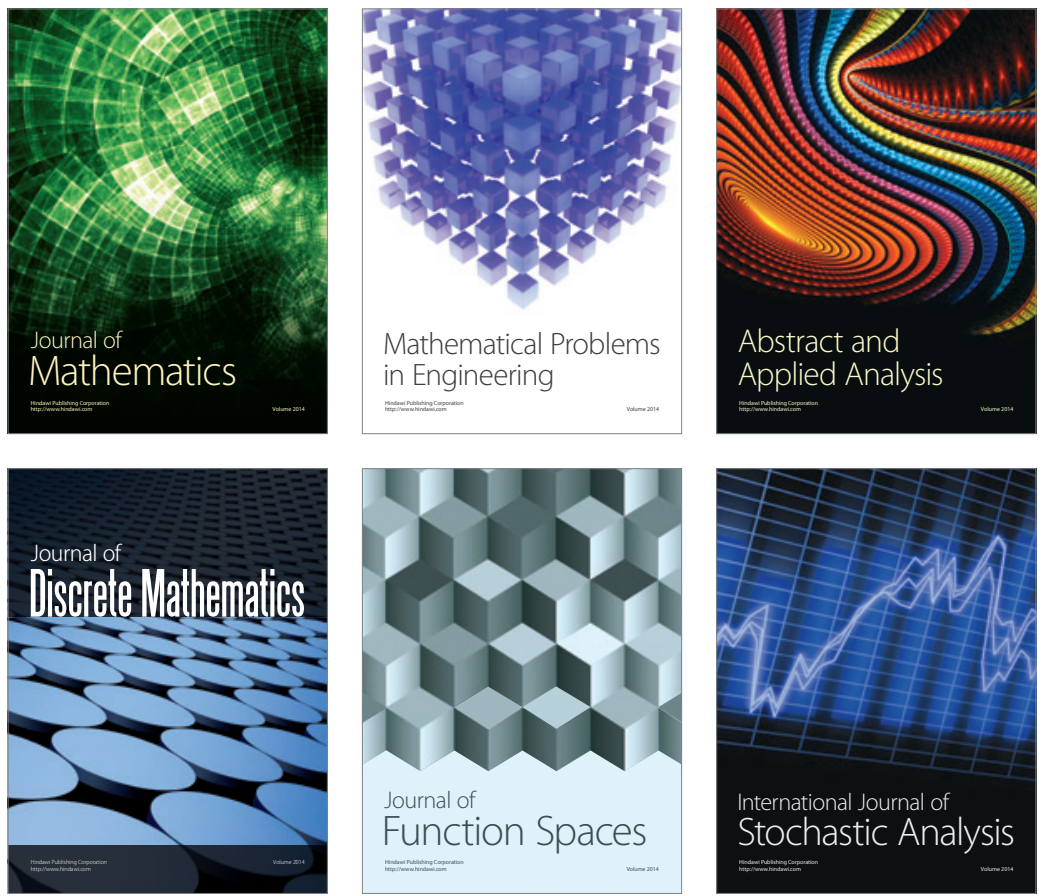

Journal of

Function Spaces

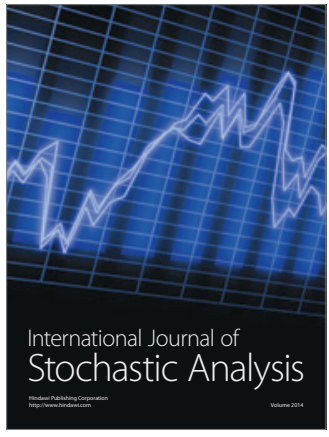

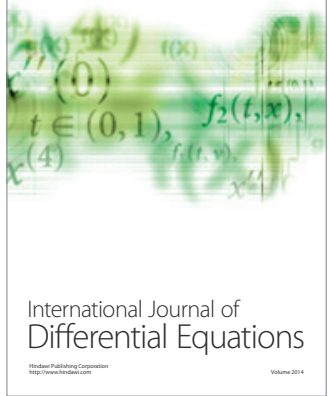
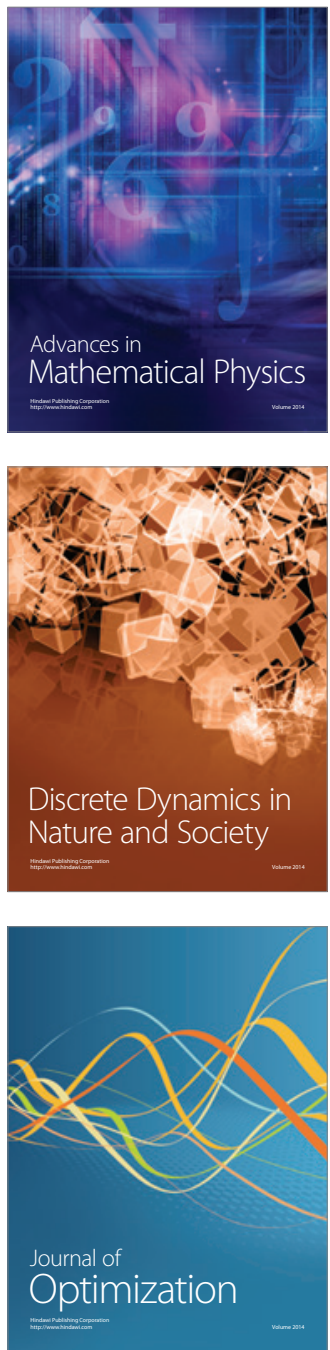